\title{
High-throughput phagocytosis assay utilizing a pH-sensitive fluorescent dye
}

\author{
Anton Beletskii ${ }^{1}$, Michael Cooper ${ }^{2}$, Priya Sriraman ${ }^{3}$, Camelia Chiriac ${ }^{3}$, Lihong Zhao ${ }^{3}$, \\ Stewart Abbot ${ }^{1}$, and Liming Yu ${ }^{1}$
}

BioTechniques 39:894-897 (December 2005)

doi 10.2144/000112001

\begin{abstract}
We describe a development of a novel high-throughput phagocytosis assay based on a $\mathrm{pH}$-sensitive cyanine dye, CypHer5ETM, which is maximally fluorescent in an acidic environment. This dye is ideally suited for the study of phagocytosis because of the acidic conditions generated in the intracellular phagocytic vesicles after particle uptake. Use of CypHer5E-labeled particles results in greatly reduced background from noninternalized particles and makes the assay more robust. Additionally, CypHer5E-labeled particles are resistant to fluorescence quenching observed in the aggressive and acidic environment of the phagosome with traditional dyes. The CypHer5E-based assay has been shown to work reliably in a variety of cell types, including primary human monocytes, primary human dendritic cells, primary human endothelial cells, human monocytic THP-1 cell line, and human/mouse hybrid macrophage cell line WBC264-9C. Inhibition of CypHer5E bead uptake by cytochalasin D was studied, and the 50\% inhibition concentration $\left(I C_{50}\right)$ was determined. The assay was performed in 96- and 384-well formats, and it is appropriate for high-throughput cellular screening of processes and compounds affecting phagocytosis. The CypHer5E phagocytosis assay is superior to existing protocols because it allows easy distinction of true phagocytosis from particle adherence and can be used in microscopy-based measurement of phagocytosis.
\end{abstract}

\section{INTRODUCTION}

Phagocytosis is an important physiological process by which macrophages, neutrophils, and other cells actively ingest and destroy invading foreign microorganisms as well as apoptotic cells and abnormal particles within the body. Aberrant phagocytosis has been implicated in the pathology of multiple sclerosis, Alzheimer disease, and atherosclerosis (1-3). Deficient phagocytosis leads to severe and recurrent bacterial and fungal infections in affected individuals (4). Moreover, it is increasingly advisable to counterscreen new immunomodulatory drugs for effects on phagocytosis.

The most widely used experimental targets for phagocytosis assays include latex beads, zymosan, and bacteria (5). Traditionally test particles are labeled with fluorescein or rhodamine dyes for detection by microscopy or by flow cytometry. Two major deficiencies associated with the use of traditional dyes are the difficulty in distinguishing adherent from ingested particles and the quenching of fluorescent labels in the acidic environment of the phagosome (5). In an attempt to overcome these deficiencies, recent techniques have been developed to distinguish extracellular and intracellular particles by using plasma membrane-impermeant quenching agents, such as ethidium bromide, trypan blue, and dye-specific antibodies, which selectively quench extracellular fluorescence $(5,6)$. Unfortunately, this approach is largely incompatible with flow cytometry applications for measurement of phagocytosis; and more importantly, general uptake of ethidium bromide by the cells makes interpretation of the results more difficult (6). Additionally, $\mathrm{pH}$-sensitive fluorescence dyes have been employed extensively in phagocytosis research, but in most cases their use is associated with spectral shifts and decreases in fluorescence intensity at the acidic $\mathrm{pH}$ found within phagosomes (5-8). We have improved upon existing methods and developed a novel labeling approach for studying phagocytosis using the $\mathrm{pH}$-sensitive cyanine dye CypHer5E $\mathrm{E}^{\mathrm{TM}}$ (9), which is minimally fluorescent at neutral $\mathrm{pH}$ outside the cell, but highly fluorescent in the acidic environment of the phagosome [pKa approximately 7.3; excitation peak $650 \mathrm{~nm} /$ emission peak $670 \mathrm{~nm}$ (9)]. Cypher5E can be used to label all standard test particles while overcoming the disadvantages associated with the use of traditional dyes.

\section{MATERIALS AND METHODS}

\section{CypHer5E Conjugation to Target Particles}

CypHer5E mono N-hydroxysuccinimide (NHS) ester was obtained from GE Healthcare (formerly Amersham Biosci-

${ }^{1}$ GE Global Research Center, Niskayuna, NY, USA, ${ }^{2}$ GE Healthcare, The Maynard Centre, Cardiff, UK, and ${ }^{3}$ GE Healthcare Discovery Systems, Piscataway, NJ, USA 
ences, Piscataway, NJ, USA). One- and three-micron Polybead Amino latex particles were purchased from PolySciences (Warrington, PA, USA), and zymosan was obtained from Sigma (St. Louis, MO, USA). For labeling, $0.5 \mathrm{~mL} 1$ - or 3- $\mu \mathrm{m}$ latex particles was spun down at $3000 \times g$ for $5 \mathrm{~min}$ and resuspended in $0.5 \mathrm{~mL} 0.1 \mathrm{M}$ sodium carbonate $(\mathrm{pH} 9.0)$, which resulted in a final concentration of $1.6 \times 10^{9}$ particles $/ \mathrm{mL}$ or $4.5 \times 10^{10}$ particles $/ \mathrm{mL}$ for 1 - and $3-\mu \mathrm{m}$ particles, respectively. Dried zymosan was resuspended in $0.1 \mathrm{M}$ sodium carbonate (pH 9.0) at a final concentration of $10 \mathrm{mg} / \mathrm{mL}$. CypHer5E mono NHS ester was dissolved in $0.1 \mathrm{M}$ sodium carbonate (pH 9.0) at $100 \mathrm{mg} / \mathrm{mL}$. The labeling reaction for the latex particles was initiated by adding $0.3 \mathrm{mg}$ CypHer5E mono NHS ester to the $0.5 \mathrm{~mL}$ particle suspension. For zymosan, $0.05 \mathrm{mg}$ CypHer5E mono NHS ester was added to $10 \mathrm{mg}$ suspended zymosan. The mixtures were then allowed to react for $2 \mathrm{~h}$ at room temperature with constant agitation. Labeled particles were removed from the reaction mixture by centrifugation at $3000 \times g(300 \times g$ for zymosan). Particles were resuspended in $20 \mathrm{~mL}$ phosphate-buffered saline (PBS; Invitrogen, Carlsbad, CA, USA) and washed again by centrifugation three more times. The final pellet was resuspended in PBS and kept
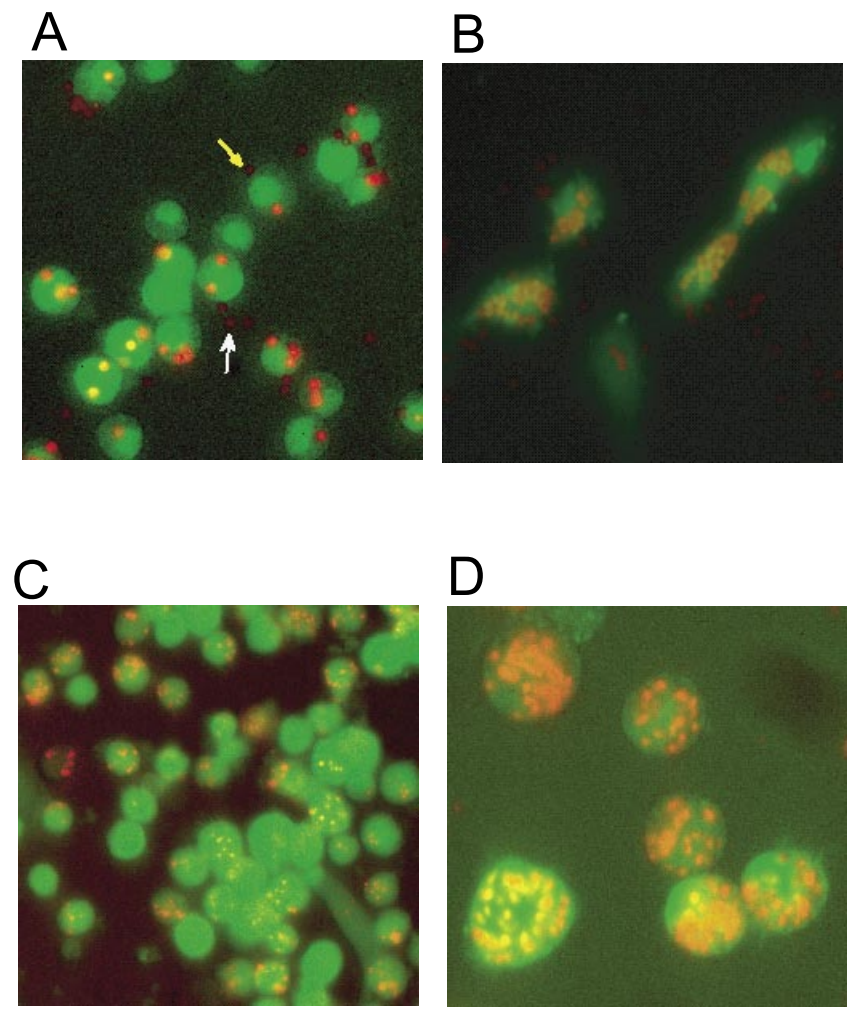

Figure 1. Phagocytosis of CypHer5E-labeled particles. (A) Phagocytosis of 3- $\mu \mathrm{m}$ CypHer5E latex beads by WBC264-9C macrophages. About 26 highly fluorescent phagocytosed particles can be seen in the image as well as 21 dimly fluorescent particles in the medium (white arrow) and adherent particles (yellow arrow). (B) Phagocytosis of 3- $\mu \mathrm{m}$ CypHer5E latex beads by human umbilical vein endothelial cells (HUVECs). (C) Phagocytosis of $1-\mu \mathrm{m}$ CypHer5E latex beads by primary human monocyte-derived immature dendritic cells. (D) Phagocytosis of CypHer5E zymosan particles by WBC264-9C macrophages. All cells were stained with Cell Tracker Green before the assay and incubated with the beads for $16 \mathrm{~h}$ before imaging. Images were acquired using IN Cell Analyzer 1000 or 3000 with a $10 \times$ objective $(\mathrm{A}-\mathrm{C})$ or a $20 \times$ objective $(\mathrm{D})$. at $4{ }^{\circ} \mathrm{C}$. Particle preparation was then tested microscopically to assure that background is no brighter than a control field with no CypHer5E present.

\section{Phagocytosis Assays}

THP-1 and WBC264-9C cell lines were from ATCC (Manassas, VA, USA). Human umbilical vein endothelial cells (HUVECs) were from Cambrex (Walkersville, MD, USA). Primary human monocytes and neutrophils were isolated from heparinized whole blood obtained by venipuncture, using Dextran 500 (GE Healthcare) sedimentation and discontinuous Ficoll $^{\circledR}$ (GE Healthcare) gradients as described (10), and used within $4 \mathrm{~h}$. Monocytes were further purified using MACS $^{\circledR}$ Human Monocyte Isolation Kit II (Miltenyi Biotec, Auburn, CA, USA). Immature dendritic cells (DCs) were derived from human blood monocytes using granulocyte macrophage colony-stimulating factor (GM-CSF)/interleukin 4 (IL-4) treatment protocol as described previously (10). Phagocytes were labeled with Cell Tracker ${ }^{\mathrm{TM}}$ Green (Invitrogen) as a cellspecific marker and used in a 96-well format.

To assure opsonization of target particles, all phagocytosis assays were conducted in the presence of $10 \%$ fetal bovine serum (FBS; Hyclone, Logan, UT, USA). Additionally, in some experiments, labeled particles were preopsonized by incubation at $37^{\circ} \mathrm{C}$ for $1 \mathrm{~h}$ with $5 \mathrm{mg} / \mathrm{mL}$ human immunoglobulin G (IgG; Sigma) or with fresh FBS followed by a wash and suspension in PBS (see the Results and Discussion section). To induce phagocytosis, particles were added to wells of a 96-well plate containing cells and subjected to a 1 -min centrifugation at $300 \times g$ to promote interaction between cells and particles. In a typical experiment, 20,000 phagocytes were mixed with 2- to 10-fold excess of CypHer5E-conjugated particles and incubated 1 to $18 \mathrm{~h}$ in $150 \mu \mathrm{L}$ RPMI 1640 medium containing 10\% FBS (Invitrogen). Cytochalasin D, genistein, and piceatannol (all from Sigma) were added as phagocytosis inhibitors when necessary and preincubated with cells for $30 \mathrm{~min}$ before the addition of beads.

\section{Microscopy and Image Analysis}

Cellular imaging was performed on IN Cell Analyzer 1000 and 3000 (GE Healthcare) using a $10 \times$ or $20 \times$ objective and fluorescein isothiocyanate (FITC)/CyTM5 excitation/emission filters. Analysis was performed using Granularity Analysis and Object Intensity Analysis Modules within the IN Cell Analysis system software suite.

\section{RESULTS AND DISCUSSION}

To set up the CypHer5E-based phagocytosis assay, we conjugated CypHer5E mono NHS ester to 1- and 3- $\mu \mathrm{m}$ latex particles and zymosan. CypHer5E mono NHS ester will label any compounds or particles containing free amino groups and may be used to label all standard particles used in phagocytosis research. In addition to phagocytosis targets, we successfully used CypHer5E labeling to study the uptake of 10-nm nanoparticles and low-density lipoprotein through endocytosis (A. Beletskii and L.Yu, unpublished). 
To assure efficient uptake of labeled particles, all phagocytosis assays were conducted in the presence of $10 \%$ FBS. We found that precoating of particles with human IgG or FBS (see the Materials and Methods section) had little effect on their uptake, and the presence of $10 \%$ FBS during the assay was sufficient for efficient phagocytosis of particles. We found that over a 32-fold range of concentrations (0.375-12 particles/ cell), the uptake was very efficient, and after overnight incubation, most of the particles were internalized.

Phagocytosed CypHer5E-conjugated particles exhibited a large increase in fluorescence intensity compared with adherent beads and beads in the medium (Figure 1, A-D). Time course studies (results not shown) indicated that increased fluorescence associated with particle phagocytosis was observable about $30 \mathrm{~min}$ after the start of the phagocytosis reaction. Using IN Cell Analyzer 1000 and 3000 systems for detection, 3- $\mu \mathrm{m}$ latex beads showed a 2- to 6-fold increase in fluorescence after ingestion compared with $3-\mu \mathrm{m}$ beads in the media (Figure 1, A and B), while 1- $\mu$ m latex beads and zymosan particles were not detectable in the media or when adherent to cells (Figure 1, C and D). Extremely low background from uningested particles is highly advantageous and translates into high assay robustness.
We have demonstrated that CypHer5E-labeled particles can be used to study phagocytosis in a wide variety of cell types including primary human monocytes (not shown) and primary human immature dendritic cells (Figure 1C). We extended previous reports that primary HUVECs can internalize zymosan particles (11) by demonstrating the phagocytosis of 3- $\mu \mathrm{m}$ CypHer5E latex beads (Figure 1B). The human monocytic THP-1 cell line exhibited robust phagocytosis of CypHer5E beads after an overnight treatment with 10 nM phorbol ester (phorbol myristate acetate; PMA) (data available upon request). While we observed phagocytosis of the CypHer5E beads by primary human neutrophils, the difference in fluorescence intensity between internalized particles and particles in the medium was minimal. This can be attributed to a greatly decreased acidity of phagosomes formed by neutrophils compared with phagosomes formed by other phagocytic cells (8).

We determined that two commonly available image analysis principles, also included within the IN Cell 3000 software suite, may be used to analyze phagocytosis of CypHer5Econjugated particles by fluorescent microscopy. Granularity analysis quantitates red phagocytosed beads (granules) within the area marked by cell-specific dye and yields normalized
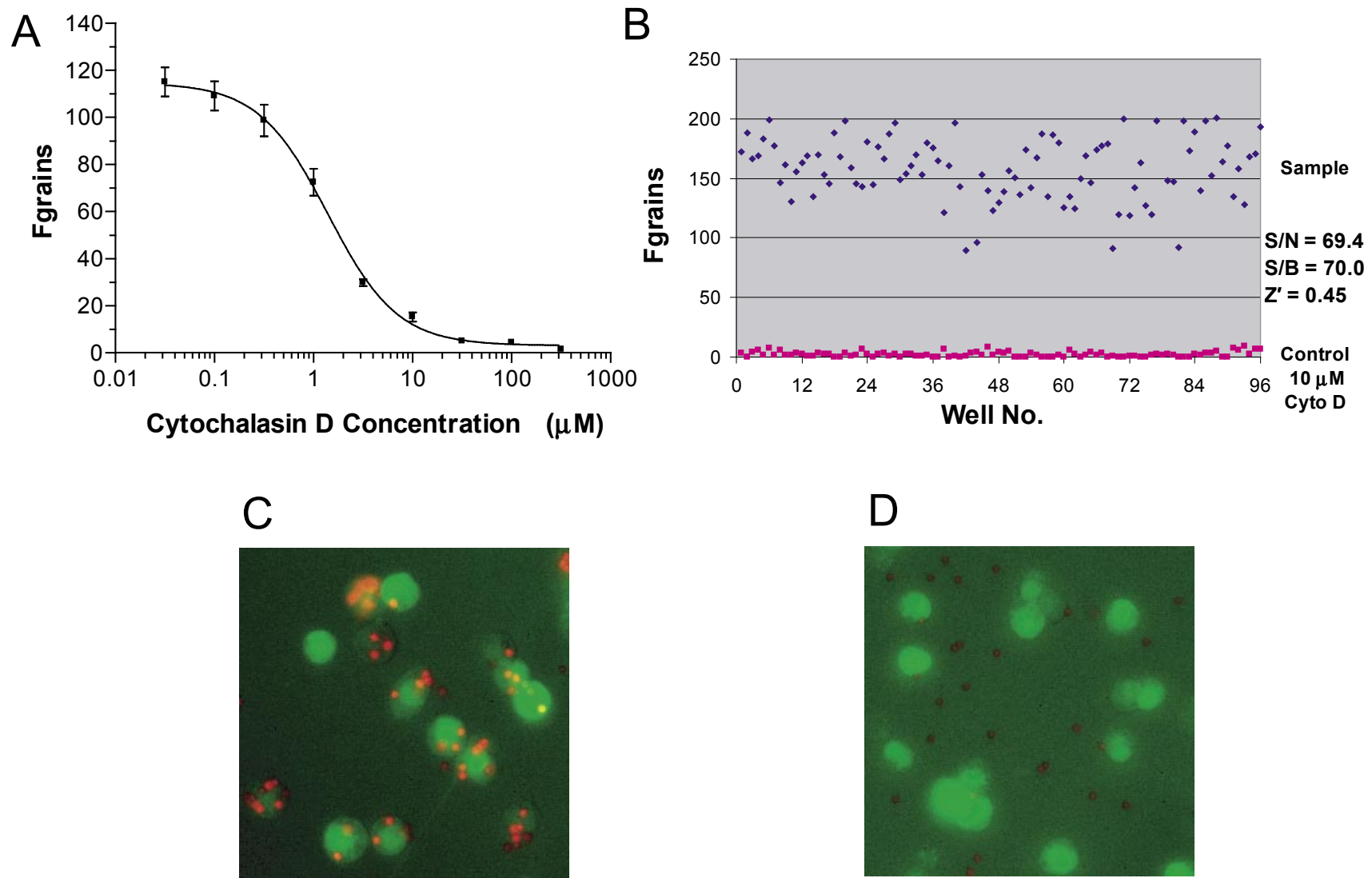

Figure 2. Analysis of phagocytosis inhibition using CypHer5E latex beads. (A) WBC264-9C macrophage cell line was incubated with 3- $\mu \mathrm{m}$ CypHer5E beads for $18 \mathrm{~h}$ in the presence of the indicated concentrations of cytochalasin D. Six wells were analyzed for each point. (B-D) WBC264-9C macrophage cell line was incubated with 3- $\mu \mathrm{m}$ CypHer5E beads in a 384-well format for $18 \mathrm{~h}$ without cytochalasin D (sample, 96 wells) or with $10 \mu \mathrm{M}$ cytochalasin D (control, 96 wells) (B). Representative images of sample (panel C, no cytochalasin D) and control (panel D, $10 \mu \mathrm{M}$ cytochalasin D added). Imaging was performed on an IN Cell Analyzer 3000 system, and results were analyzed using the Granularity Analysis module for the IN Cell Analyzer 3000. Fgrains, normalized measure of the fraction of cellular fluorescence present in the qualifying beads (grains); S/N, signal-to-noise (13); S/B, signal-to-background (13); Z', Z-factor (13); Cyto D, cytochalasin D. 
data. Object intensity analysis counts highly fluorescent beads inside the phagosomes, filters out weakly fluorescent beads in the medium, and does not require cell staining. Depending on the need to normalize data or the availability of cell staining, either principle can be used to measure the extent of CypHer5E-conjugated particle phagocytosis. The granularity analysis module for the IN Cell Analyzer 3000 was used to measure inhibition of 3- $\mu \mathrm{m}$ CypHer5E bead phagocytosis by cytochalasin D in WBC264-9C macrophages to determine if CypHer5E-conjugated beads could be used for the screening of compounds affecting phagocytosis (Figure 2A). A cytochalasin D concentration of $1.4 \mu \mathrm{M}$ is required to inhibit the phagocytosis level to $50 \%$ of the uninhibited response (the $50 \%$ inhibition concentration; $\left.\mathrm{IC}_{50}\right)$, which agrees well with the published values (12). Similar experiments were performed with the tyrosine kinase inhibitor genistein and the Syk-family kinase inhibitor piceatannol, and inhibition of phagocytosis was observed with an $\mathrm{IC}_{50}$ of approximately 120 $\mu \mathrm{M}$ for both compounds in the WBC264-9E cell line.

To demonstrate that CypHer5E-labeled particles can be used for high-throughput screening, we determined the Zfactor (13) by treating 96 wells of a 384-well plate containing WBC264-9C macrophages with $10 \mu \mathrm{M}$ cytochalasin D while leaving another 96 wells untreated (Figure 2B). The Z-factor is reflective of both the assay signal dynamic range and the data variation associated with the signal measurements and, therefore, is suitable for assay quality assessment (13). As can be seen from Figure 2B, a Z-factor of 0.45 for the assay described here approaches the value of 0.5 , commonly considered acceptable for high-throughput cellular screening, and is well above the minimum of $0.2-0.3$ required for meaningful cell-based assays.

In conclusion, we have presented a simple and convenient assay for studying phagocytosis. The fluorescent signal generated by internalized CypHer5E-labeled particles is easily distinguishable from the background of noninternalized particles, which results in increased assay robustness. Additionally, CypHer5E-labeled particles are resistant to fluorescence quenching observed in the aggressive and acidic environment of the phagosome with the traditional dyes (5). However, application of the assay in neutrophils is limited due to decreased acidity of phagosomes formed by neutrophils compromising internalized particle fluorescence intensity. The assay is superior to existing protocols, because it allows the easy differentiation of true phagocytosis from particle adherence in microscopy-based measurements of phagocytosis and potentially can also be used in flow cytometry-based assays.

\section{ACKNOWLEDGMENTS}

We thank Dr. Fergus R. McKenzie for the original idea and preliminary work on this project, Dr. Christoph Hergersberg for support and encouragement, and Sian Godwin for critical reading of the manuscript. The authors would like to apologize to many researchers whose excellent work on the development of the current phagocytosis assays was not cited due to space limitations.

\section{COMPETING INTERESTS STATEMENT}

The authors are employees of GE Healthcare, which manufactures CypHer5E dye.

\section{REFERENCES}

1. van der Laan, L.J., S.R. Ruuls, K.S. Weber, I.J. Lodder, E.A. Dopp, and C.D. Dijkstra. 1996. Macrophage phagocytosis of myelin in vitro determined by flow cytometry: phagocytosis is mediated by CR 3 and induces production of tumor necrosis factor-alpha and nitric oxide. J. Neuroimmunol. 70:145-152.

2. Koenigsknecht, J. and G. Landreth. 2004. Microglial phagocytosis of fibrillar beta-amyloid through a beta1 integrin-dependent mechanism. J. Neurosci. 24:9838-9846.

3. Patel, M., J. Morrow, F.R. Maxfield, D.K. Strickland, S. Greenberg, and I. Tabas. 2003. The cytoplasmic domain of the low density lipoprotein (LDL) receptor-related protein, but not that of the LDL receptor, triggers phagocytosis. J. Biol. Chem. 278:44799-44807.

4. Rotrosen, D. and J.I. Gallin. 1987. Disorders of phagocyte function. Annu. Rev. Immunol. 5:127-150.

5. Chow, C.W., G.P. Downey, and S. Grinstein. 2004. Measurement of phagocytosis and phagosomal maturation, p. 15.7.1-15.7.33. In J.S. Bonifacino, M. Dasso, J. Lippincott-Schwartz, J.B. Harford, and K.M. Yamada (Eds.), Current Protocols in Cell Biology. John Wiley \& Sons, New York.

6. Drevets, D.A. and P.A. Campbell. 1991. Macrophage phagocytosis: use of fluorescence microscopy to distinguish between extracellular and intracellular bacteria. J. Immunol. Methods 142:31-38.

7. Schramm, N., C.R. Bagnell, and P.B. Wyrick. 1996. Vesicles containing Chlamydia trachomatis serovar L2 remain above $\mathrm{pH} 6$ within HEC-1B cells. Infect. Immun. 64:1208-1214.

8. Jankowski, A., C.C. Scott, and S. Grinstein. 2002. Determinants of the phagosomal pH in neutrophils. J. Biol. Chem. 277:6059-6066.

9. Adie, E.J., S. Kalinka, L. Smith, M.J. Francis, A. Marenghi, M.E. Cooper, M. Briggs, N.P. Michael, et al. 2002 A pH-sensitive fluor, CypHer 5 , used to monitor agonist-induced $\mathrm{G}$ protein-coupled receptor internalization in live cells. BioTechniques 33:1152-1154.

10.Biddison, W.E. 1998. Preparation and culture of human lymphocytes, $\mathrm{p}$ 2.2.1-2.2.13. In J.S. Bonifacino, M. Dasso, J. Lippincott-Schwartz, J.B Harford, and K.M. Yamada (Eds.), Current Protocols in Cell Biology. John Wiley \& Sons, New York.

11.Langeggen, H., E. Namork, E. Johnson, and G. Hetland. 2003. HUVEC take up opsonized zymosan particles and secrete cytokines IL-6 and IL-8 in vitro. FEMS Immunol. Med. Microbiol. 36:55-61.

12.Sakr, S.W., R.J. Eddy, H. Barth, F. Wang, S. Greenberg, F.R. Maxfield, and I. Tabas. 2001. The uptake and degradation of matrix-bound lipoproteins by macrophages require an intact actin cytoskeleton, Rho family GTPases, and myosin ATPase activity. J. Biol. Chem. 276:37649-37658.

13.Zhang, J.H., T.D. Chung, and K.R. Oldenburg. 1999. A simple statistical parameter for use in evaluation and validation of high throughput screening assays. J. Biomol. Screen. 4:67-73.

Address correspondence to Anton Beletskii, GE Global Research Center, 1 Research Circle, Bldg. K-1, Room 5D39, Niskayuna, NY 12309, USA.e-mail: anton.beletskii@ge.com

To purchase reprints

of this article, contact

Reprints@BioTechniques.com 